\title{
Interactive comment on "The firn meltwater Retention Model Intercomparison Project (RetMIP): Evaluation of nine firn models at four weather station sites on the Greenland ice sheet" by Baptiste Vandecrux et al.
}

\author{
Samuel Morin (Referee) \\ samuel.morin@meteo.fr \\ Received and published: 7 May 2020
}

The manuscript by Vandecrux et al., entitled "The firn meltwater Retention Model Intercomparison Project (RetMIP): Evaluation of nine firn models at four weather station sites on the Greenland ice sheet", provides results from a recent intercomparison of firn models dedicated to their handling of meltwater retention and runoff. This topic has attracted a lot of interest over the past decade, following the discovery of subwater transfer is a critical, yet very complex process governing the energy and mass 
balance of firn below the surface. The manuscript undoubtedly fits the scope of The Cryosphere. It is generally well designed and easy to understand, although, like many other manuscripts reporting on intercomparisons, the reading can be a bit cumbersome and the results will mostly appeal to experts in this field. I recommend publication of this article, and I provide here below some feedback and suggestions, which may be considered by the authors in case they lead to improvements of the manuscript.

Page 1, line 1-2: "Perennial snow, or firn, covers $80 \%$ of the Greenland ice sheet and has the capacity to retain part of the surface meltwater, buffering the ice sheet's contribution to sea level". This sentence could in fact be quite misleading and I suggest reformulating, either at least acknowledging that the "buffering" acts upon sea level "rise*, or maybe more generally, leave out the term "buffering" (which could be inappropriate in some cases where runoff generation can increase the contribution of the ice sheet to sea level rise), and use a more neutral term referring to the fact that firn processes influence the behaviour of the ice sheet and affect its contribution to sea level rise.

Page 1, line 3 : "weather-station-derived" : maybe consider unpacking the wording, this is quite tedious to read.

Page 2, line 60 : I think it could be useful, and appeal to a wider community of readers, if this article could provide more information on what a "firn" model is, or what a "snow and firn" model is if the two terms here are meant to be combined. Indeed, GCMs and RCMs all feature a "snow" component in their land surface models, and many such models do not handle ice sheets differently from a long-lasting seasonal snowpack. It could thus be quite appropriate to provide more background on how snow and firn processes are considered in GCMs and RCMs (or other tools used for reanalyses etc.), in order to make this effort even more useful in a CMIP6/IPCC context. This could make it possible to establish how the firn models used for this intercomparison fit in this wider context, thereby providing information on whether the results of this intercomparison are relevant when discussing the results of existing GCMs and RCMs projection. Also

Printer-friendly version

Discussion paper 
(see below point too), it would be good to introduce what are the typical input/output to firn models, because they seem to deviate from typical input/output of land surface models, hence the link to GCMs/RCMs/etc. is not direct.

Page 2, line 64 : Although I know this can be debated, I have strong personal reservations against the term "validation", which I believe is beyond reach in any geosciences field because the "truth" is never known hence "validation" (in the strong sense) can only be elusive. I much prefer the term "evaluation", which better encapsulates the fact that the evaluation results from a comparison with observations, which also carry some uncertainties. I was happy to see the term "evaluation" in the title and in the abstract, so it was disappointing to see the term "validation" popping up in the introduction. Maybe this can be harmonized throughout. Along the same line, in several instances the comparison between model output and observations is referred as a "bias". Here again, "bias" is a term which includes some judgement of value, implicitly assuming that observations are the "truth". I think that observations are never the "truth" and, especially regarding in-situ snow and firn observations, we know that observations are intrinsically prone to significant errors, in addition to large spatial variability for many variables at all scales, which induces representativeness issues. In this context, I much prefer referring to "deviations" between model results and observations, without using the term bias. I note that the term "deviation" is used in several places in the manuscript, including in figure captions (e.g. caption of Figure 6, which I think is perfectly worded), so maybe this could be harmonized in the text.

Page 2, line 67 : Here is a good example where more information could be provided on whether (some of) the firn models included in this intercomparison are representative to how firn processes are handled in GCM/RCM/NWP models, so that the results can be used to analyse some of the output of such models. At present, and even though some of the information is provided in section 2 , the models included in the intercomparison are not categorized explicitly according to their use, and I think it could be helpful to the scientific community to provide the rationale and the results of the intercomparison in

Printer-friendly version

Discussion paper 
a way that can (somehow) be transferred to the interpretation of other model results.

Page 3, line 91 : it seems that there is a typo in this reference, I believe this should be 1998. I haven't checked all reference, but if they were typed by hand and not using literature management software, there may be other errors in the references.

Page 4, line 1: This table is very useful, I suggest adding a column for providing the extended name (developing the acronym) and, more to the point, adding a column on how the model is typically used (included/coupled to a land surface model in $\mathrm{RCM} / \mathrm{GCM} / \mathrm{NPW}$ context, or purely offline for process investigations etc.). I'm convinced that the authors can easily define several categories within which models can be classified (these categories could even be used in the results and discussion, if common features, or not, emerge from these various categories, in addition to discussing results referring to process representation in models).

Page 4, Line 98 : I think it would be good to spell out the acronyms in the titles of subsections $2.1,2.2$ etc., this is otherwise quite obscure for non-expert readers not accustomed to the acronyms of these firn models.

Page 5, line 116 : extra "“- between ASIRAS and instrument

Page 5, line 131 : it seems to me that layers defined by a w.e. (e.g. mass per unit surface area) should not be qualified by a "thickness", which refers to a distance (in $\mathrm{m}$ ). If the model is formulated in terms of layers with a given mass, then I believe the text should refer to this, and the substitution of "thickness" with "mass" will accurately represent how the model is formulated.

Page 7, Table 2 : I think that the column on "Hydraulic conductivity" needs some attention. The van Genuchten (1980) article provides a way to link between the saturated hydraulic conductivity and the hydraulic conductivity, which for snow has been addressed by several studies such as Shimizu (1970), Calonne et al. (2014) or can be used using geometrical estimates such as Carman-Kozeny (see Calonne et al., 2014 
for a review of existing formulations, and Wever et al., 2014, for context). Hence I suggest to double check, for each model, what is the parameterization used for estimating the saturated hydraulic conductivity (corresponding to the permeability) from the microstructure (density, specific surface area/grain size) and the formulation used to derive the hydraulic conductivity (van Genuchten (1980) is probably widely used). This column seems to be lumping and mixing the two.

Interactive

Page 8, line 177 : I'm not fully convinced by the formulation of how the forcing data are introduced. "Any bias in forcing data propagates into the model output" : I'd rather suggest that any *difference* in forcing data propagates into differences in model outputs. The term "bias" is here inappropriate I think (see comments above). Further, "To make sure we compare and evaluate the models independently of biases that may exist in forcing datasets that come from RCMs, we use meteorological fields derived from five weather stations at four sites." I think this sentence needs rephrasing, because it gives the impression that only RCM atmospheric fields can be biases, and in-situ atmospheric observations are not biased. I don't see the point in referred to RCM here at all, but simply state that "To make sure we compare and evaluate the models independently of differences due to forcing data, we use for all models the same meteorological fields derived from five weather stations at four sites." The references to RCM data is absolutely not needed here, and in the current formulations I consider it misleading and improper.

Page 9, line 202 : If I understand well, the firn models are driven by 3-hourly skin temperature, meltwater generation (what it this ?) and net snow accumulation. I think this warrants an explicit statement on the forcing data for firn models (see my comment regarding the introduction), because this appears to be quite different from forcing data of land surface models (including the snow component), usually driven by air temperature, relative humidity, incoming shortwave radiation, incoming longwave radiation, wind speed (and direction) and snowfall and rainfall rate (in offline or online applications). In this context, it would be good to quickly introduce how the firn model

Printer-friendly version

Discussion paper 
data input are typically computed within GCM/RCM/NWP models where they are implemented. I also think that it would be beneficial for the manuscript, if the material provided in the Supplement regarding how the models were adapted in order to contribute to the intercomparison, could be placed within the body of the article. [In fact, I'm in favour of moving the whole supplementary material into the article, which is quite technical anyway, and which I think would benefit from having all the information in the same document].

Page 10, Table 3 : Mean annual air temperature data should probably be homogenized in terms of formatting (why is the number provided by $0.1^{\circ} \mathrm{C}$ resolution of KAN_U and rounded to the unit ${ }^{\circ} \mathrm{C}$ for other sites ?)

Page 20, line 371 : Eulerian

Page 20, line 384 : it seems that the sentence does not end as planned. Was it the intent to refer to Calonne et al. (2019; https://doi.org/10.1029/2019GL085228 ) which may provide some hints into how to parameterize firn thermal conductivity ?

Page 21, line 404 : The last sentence "Especially, the heterogeneous nature of the firn, the presence of vertical ice features in the firn, the variability in surface snow density/thermal conductivity as well as firn ventilation are processes not currently included in the models and should be subject of future research." is certainly true but is quite vague. Based on existing literature (e.g. Albert et al.) is it possible to elaborate more on the expected direction of change and whether accounting for such processes would be beneficial (and at what numerical cost) ?

Page 21, line 413 : add "with" after "match"

Page 22, line 433 : on the "fresh snow" density issues and beyond, there is little discussion in the manuscript on the connection between snow cover models (such as those embedded in land surface schemes) and firn models. Wouldn't it be adequate that surface processes are handlded by snow cover model rather than firn models, taking

Printer-friendly version

Discussion paper 
advantage of the features of both types of models ? This connects with the question on how firn models are used within coupled GCM/RCM or offline (see comments anove).

Page 22, line 443 : It should be mentioned here that the uncertainty envelope corresponds to $+/-2$ standard deviation (+/- 2 sigma). This information is missing here (although it is provided later in the conclusion). Regarding the temperature, I see a limitation to the estimate provided here, in the sense that the temperature of the firn cannot exceed $0^{\circ} \mathrm{C}$. How does this impact the uncertainty range provided for temperature?

Page 23, line 451 : What is the argument for stating that this uncertainty range applies in situations where observations are not available?

Page 24, line 482 : "much faster drainage of the aquifer" : faster than what ?

Page 24, line 484 : A qualifier is probably missing before "models": "existing firn models" ? "firn models considered in this intercomparison" ?

Page 24, line 490 : I suggest not using the term "reality" in scientific publications. Furthermore, a reference is missing to support the statement in this sentence.

Figures : I didn't notice any major flaw in the design or content of the figures, which are appropriate to convey the results of this intercomparison.

Interactive comment on The Cryosphere Discuss., https://doi.org/10.5194/tc-2019-331, 2020. 